\title{
Effect of neodymium concentration on structural and optical properties of tellurite based glass system
}

\begin{abstract}
Neodymium doped zinc borotellurite glass system were fabricated by using conventional melt-quenching method. The structural properties of the glass system were characterized by using X-ray Diffraction (XRD) method and Fourier Transform analysis (FTIR). The amorphous nature of the glass system was confirmed by using X-ray diffraction method. The transmission band of $\mathrm{TeO} 3$ structural units which indicate the existence of non-bridging oxygen was shown by FTIR analysis. The optical properties of the glass system were determined by using UV-Vis spectrophotometer. Several bands were shown in the absorption spectra which indicate the characteristic of neodymium ions. The obtained values of indirect optical band gap, Eopt lies in the range of $3.151 \mathrm{eV}$ and $3.184 \mathrm{eV}$.
\end{abstract}

Keyword: Fourier transform infrared spectroscopy (FTIR); Optical band gap; Optical materials 